\title{
Observational constraint on the interacting dark energy models including the Sandage-Loeb test
}

\author{
Ming-Jian Zhang ${ }^{\mathrm{a}}$, Wen-Biao Liu ${ }^{\mathrm{b}}$ \\ Department of Physics, Institute of Theoretical Physics, Beijing Normal University, Beijing 100875, China
}

Received: 6 February 2014 / Accepted: 14 April 2014 / Published online: 1 May 2014

(C) The Author(s) 2014. This article is published with open access at Springerlink.com

\begin{abstract}
Two types of interacting dark energy models are investigated using the type Ia supernova (SNIa), observational $H(z)$ data (OHD), cosmic microwave background shift parameter, and the secular Sandage-Loeb (SL) test. In the investigation, we have used two sets of parameter priors including WMAP-9 and Planck 2013. They have shown some interesting differences. We find that the inclusion of SL test can obviously provide a more stringent constraint on the parameters in both models. For the constant coupling model, the interaction term has been improved to be only a half of the original scale on corresponding errors. Comparing with only SNIa and OHD, we find that the inclusion of the SL test almost reduces the best-fit interaction to zero, which indicates that the higher-redshift observation including the SL test is necessary to track the evolution of the interaction. For the varying coupling model, data with the inclusion of the SL test show that the parameter $\xi$ at $1 \sigma$ C.L. in Planck priors is $\xi>3$, where the constant $\xi$ is characteristic for the severity of the coincidence problem. This indicates that the coincidence problem will be less severe. We then reconstruct the interaction $\delta(z)$, and we find that the best-fit interaction is also negative, similar to the constant coupling model. However, for a high redshift, the interaction generally vanishes at infinity. We also find that the phantom-like dark energy with $w_{X}<-1$ is favored over the $\Lambda \mathrm{CDM}$ model.
\end{abstract}

\section{Introduction}

The accelerating expansion of the universe is an extraordinary discovery of modern cosmology following Hubble's discovery of the expansion. A number of independent cosmological probes over the past decade have supported this phenomenon. Examples include observations of the type Ia supernova (SNIa) [1], the large scale structure [2], and the

\footnotetext{
a e-mail: zhangmj@mail.bnu.edu.cn

be-mail: wbliu@bnu.edu.cn
}

cosmic microwave background (CMB) anisotropy [3]. After this discovery, several theoretical attempts have been made to explain it. They generally include the dark energy, modified gravity, and the local inhomogeneous model. Among the numerous candidates of dark energy, the $\Lambda \mathrm{CDM}$ model with a cosmological constant is considered to be the simplest and most robust from the point of view of observations. However, the theoretical magnitude of this constant from the particle physical theory is about 120 orders larger than the constraint from observations. As a result, such a trivial cosmological constant falls into the entanglement of two notable problems. One is the fine-tuning problem, which asks why the observed value of cosmological constant energy density $\rho_{\Lambda}$ is so small [4,5]. The other is the coincidence problem [6] which asks why the order of the magnitude of the inappreciable cosmological constant is the same as the present matter density with the expansion of universe, i.e., $\Omega_{\Lambda 0} \sim \Omega_{m 0}$. Generally, we believe that the evolution of the cosmic component of the energy density should satisfy $\rho_{i} \propto a^{-3\left(1+w_{i}\right)}$ during the expansion of our universe, where $w_{i}$ is its equation of state and $a$ is the cosmic scale factor. Thus, the energy density of the cosmological constant with $w_{\Lambda}=-1$ should not change, while the energy density of the matter would decrease with $a^{-3}$. From the observations, however, they are comparable at the present epoch. Some approaches have been raised to reconcile this problem, such as the odd anthropic principle [7-9] and the "tracker field" model [10]. In the latter approach, dark energy is no longer a constant, but some scalar fields which are usually in the form of the quintessence [11], phantom [12], k-essence [13], as well as quintom [14] fields. Nevertheless, they cannot get rid of the suspicion of finetuning of model parameters in such models. Moreover, the nature of the dark energy is still mysterious. An interesting alternative is the interacting model which assumes an interaction between matter and dark energy. In this initial phenomenological form [15], the evolution of the dark energy density $\rho_{X}$ is assumed to follow a ratio relation, namely, $\rho_{X} \propto \rho_{m} a^{\xi}$ and $\Omega_{X} \propto \Omega_{m} a^{\xi}$, where the scaling parame- 
ter $\xi$ is a constant to mirror the severity of the coincidence problem. Especially, this model can recover to the $\Lambda \mathrm{CDM}$ and self-similar solutions [16,17] for the case $\xi=3$ and $\xi=0$, respectively. Because the interaction term in this form is redshift dependent, this model is usually called the varying coupling model. Different from the varying model, a constant coupling model with constant interaction term is also provided $[18,19]$ in which the matter density may not follow the common relationship $\rho_{m} \propto a^{-3}$. The formulas in this model are plump, such as the ones of the general type $\rho_{X} / \rho_{m}=f(a)$ [20] where $f(a)$ is a function of the scale factor $a$, or the specific interaction term models [21]. Observationally, a large amount of observational data, such as the SNIa, CMB, the baryonic acoustic oscillation (BAO), and the observational $H(z)$ data (OHD), are widely used to place constraints on these coupling models. For the constant coupling model, investigations in Refs. $[19,22]$ deem that a large coupling can change the evolution of the universe during the matter-dominated epoch. For the varying coupling model, investigations [23-25] found that SNIa and BAO data cannot provide stringent constraints on the parameter $\xi$ until inclusion of the CMB data. We note that the above observations apart from the CMB mainly focus on the redshift $z<2$. Therefore, a probe at higher redshift is necessary and is expected to better track the evolution of the universe.

In 1962, Sandage [26] proposed a promising survey named redshift drift to directly probe the dynamics of the cosmic expansion. In 1998, Loeb [27] found that this effect could be achieved by many techniques. One of them is to collect the secular variation of the expansion rate during the evolution of the universe from the wavelength shift of a quasar (QSO), Ly $\alpha$ absorption lines. Therefore, this observation is usually named the Sandage-Loeb (SL) test. According to the schedule, a new-generation European Extremely Large Telescope (E-ELT) would monitor the cosmic expansion history from the QSOs Ly $\alpha$ absorption lines in the region $z=2-5$ where other observations are inaccessible. For a complement, it is useful for us to revisit the interacting dark energy models using this test. Recently, Liske et al. [28-30] simulated some SL data using the Monte Carlo method. From previous work, we find that it generally produces excellent constraint on the cosmological models, such as the holographic dark energy [31], modified gravity models [32], new agegraphic, and Ricci dark energy models [33]. More recently, Li et al. [34] found that the SL test is able to significantly break degeneracies between model parameters of $f(R)$ modified gravity, and $f(T)$ gravity theory, when combined with the latest observations. More importantly, the SL test could identify the dark energy model with an oscillating equation of state and the models beyond general relativity with varying gravitational coupling, where the SNIa is no option [35]. In this paper, we would extend the analysis on the coupling dark energy models to a deeper redshift interval by virtue of the
SL test. Following previous work, we shall concentrate on two common interacting models: (1) a model with constant interaction term $\delta[18,19]$ and (2) a varying coupling model with term $\delta(z)$ initially proposed by Dalal et al. [15].

The paper is organized as follows. In Sect. 2, we introduce the basic equations of the phenomenological interacting models. In Sect. 3, we illustrate the corresponding observational data and constraint methods. In Sect. 4, we display the constraint result from observational data. Finally, we summarize our main conclusion and present a discussion in Sect. 5.

\section{Phenomenological interacting models}

The interacting cosmological model is an alternative way to solve the coincidence puzzle. In this paper, we will consider two fossil models with interaction between matter and dark energy, namely, the constant coupling and varying coupling models. Throughout this paper, we assume a flat FRW universe with $\Omega_{m}+\Omega_{X}=1$ and a constant equation of state (EoS) $w_{X}$ of the dark energy. The Friedmann equation under such assumptions is

$3 H^{2}=8 \pi G\left(\rho_{m}+\rho_{X}\right)$.

The conservation equations for these interacting models should read

$$
\begin{aligned}
& \dot{\rho}_{m}+3 H \rho_{m}=+\Gamma \rho_{m}, \\
& \dot{\rho}_{X}+3 H\left(\rho_{X}+p_{X}\right)=-\Gamma \rho_{m},
\end{aligned}
$$

where $H=\dot{a} / a$ is the Hubble parameter, $\Gamma$ is the interaction term. The dot denotes the derivative with respect to the cosmic time. Note that the total energy density is conserved, although the individual energy density may not obey the conservation law. For convenience, we commonly define a dimensionless interaction term

$\delta=\Gamma / H$

Generally, a positive $\delta(\delta>0)$ denotes energy transfer from dark energy to matter, while energy would be transferred from matter to dark energy for $\delta<0$.

\subsection{Constant coupling model}

For the $\Lambda \mathrm{CDM}$ model, evolution of the matter energy density should obey the relation $\rho_{m} \propto a^{-3}$. In order to reconcile the coincidence problem, the constant coupling model states that the evolution of the matter density does not always satisfy the above relation, but that there is a small modification to it. The energy density of matter in this case usually can be written as [18, 19,22]

$\rho_{m}=\rho_{m 0} a^{-3+\delta}=\rho_{m 0}(1+z)^{3-\delta}$, 
where $\rho_{m 0}$ is the matter energy density today. The parameter $\delta$, which should be constrained by the observational data, indicates a deviation of the matter density evolution from the regular relation. Assuming a constant $\operatorname{EoS} w_{X}$ of the dark energy, we obtain the energy density of the dark energy from (3) as

$$
\begin{aligned}
\rho_{X}= & \rho_{X 0}(1+z)^{3\left(1+w_{X}\right)} \\
& +\rho_{m 0} \frac{\delta}{\delta+3 w_{X}}\left[(1+z)^{3\left(1+w_{X}\right)}-(1+z)^{3-\delta}\right],
\end{aligned}
$$

where $\rho_{X 0}$ is the dark energy density today. We note that the corresponding dark energy density no longer obeys the relation $\rho_{X} \propto a^{-3\left(1+w_{X}\right)}$, and it presents a decaying component in the second term of (6). The expansion rate, therefore, can be obtained following the Friedmann equation (1) as

$$
\begin{aligned}
E^{2}(z)= & \Omega_{X 0}(1+z)^{3\left(1+w_{X}\right)} \\
& +\frac{1-\Omega_{X 0}}{\delta+3 w_{X}}\left[\delta(1+z)^{3\left(1+w_{X}\right)}+3 w_{X}(1+z)^{3-\delta}\right],
\end{aligned}
$$

where the dark energy density parameter today is $\Omega_{X 0}=$ $8 \pi G \rho_{X 0} /\left(3 H_{0}^{2}\right)$. The present matter density parameter is thus $\Omega_{m 0}=1-\Omega_{X 0}$. Based on the relationship between deceleration factor $q(z)$ and expansion rate $E(z)$, the transition redshift (where $q(z)=0$ ) from a decelerating expansion to an accelerating expansion is given by

$z_{t}=\left[\frac{3 w_{X}}{3 w_{X}+1} \frac{\left(1-\Omega_{X 0}\right)(\delta-1)}{3 w_{X} \Omega_{X 0}+\delta}\right]^{\frac{1}{3 w_{X}+\delta}}-1$.

According to the suggestion by WMAP-9 [36], we fix the present dark energy density parameter as $\Omega_{X 0}=0.724$, $w_{X}=-1.14$, and then we plot the transition redshift at different interaction terms $\delta$ in Fig. 1. As introduced in Sect. 1, accelerating expansion has been confirmed by many observations. Therefore, the transition redshift should be positive. In fact, much literature found that $z_{t}$ may be less than unity. Thus, we obtain from Fig. 1 that the interaction term should be $\delta<1$. We find that the transition redshift slowly increases with the increase of $\delta$. Interestingly, a model-independent transition redshift test can also precisely determine $\delta$. For example, as Riess et al. [37] evaluated from the SNIa at $z>1$ using the Hubble Space Telescope, the transition redshift is $z_{t}=0.46 \pm 0.13$. Then the corresponding interaction term can be estimated at $-1.21<\delta<-0.16$.

\subsection{Varying coupling model}

The varying coupling model considered in this section is the classical scenario proposed by Dalal et al. [15]. Within the underlying theoretical assumptions, the relation between dark energy and matter densities is

$\rho_{X} \propto \rho_{m} a^{\xi}, \quad \Omega_{X} \propto \Omega_{m} a^{\xi}$,

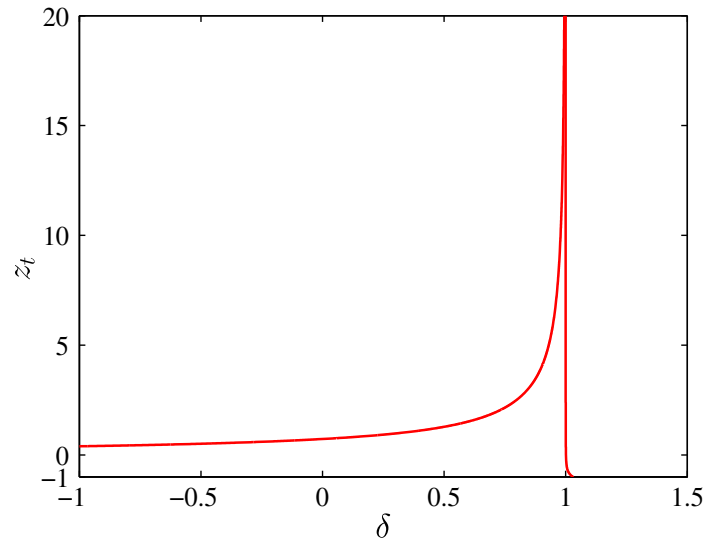

Fig. 1 Transition redshift $z_{t}$ at different interaction term $\delta$ with fixed $\Omega_{X 0}=0.724$ and $w_{X}=-1.14$ for the constant coupling model

where the constant $\xi$ characterizes the severity of the coincidence problem. Especially, this model can recover $\Lambda \mathrm{CDM}$ and self-similar solutions [16,17] for the case $\xi=3$ and $\xi=$ 0 , respectively. For the FRW universe with $\Omega_{m}+\Omega_{X}=1$, the dark energy density parameter $\Omega_{X}$ can be solved based on (9). From the conservation equations (2) and (3), we obtain the interaction term [22]

$\delta(z)=\frac{\delta_{0}}{\Omega_{X 0}+\left(1-\Omega_{X 0}\right)(1+z)^{\xi}}$,

where $\delta_{0}=-\left(\xi+3 w_{X}\right) \Omega_{X 0}$ is the interaction term today and $\Omega_{X 0}$ is the dark energy density parameter today. We note that the interaction is absent when $\xi=-3 w_{X}$, which denotes the standard cosmology. Inversely, the case $\xi \neq-3 w_{X}$ corresponds to a non-standard cosmology. With the interaction term $\delta$, the dimensionless Hubble parameter can be obtained from the Friedmann equation (1) [22]

$E^{2}(z)=(1+z)^{3}\left[1-\Omega_{X 0}+\Omega_{X 0}(1+z)^{-\xi}\right]^{-3 w_{X} / \xi}$.

The free parameters $\left(\Omega_{X 0}, \xi, w_{X}\right)$ eventually can be determined by the observational data. Following the above procedure in the constant coupling model, we can obtain the corresponding transition redshift from the deceleration factor $q(z)=0$ as

$z_{t}=\left[\frac{1-\Omega_{X 0}}{\Omega_{X 0}\left(-3 w_{X}-1\right)}\right]^{-1 / \xi}-1$.

Fixing the parameters $\Omega_{X 0}$ and $w_{X}$ suggested by the WMAP9, we plot the transition redshift for different $\xi$ in Fig. 2. We find that the positive transition redshift requires constant $\xi>0$. With the increase of $\xi$, the transition redshift generally decreases. In the following section, we will carry out the observational constraints on these coupling models. 


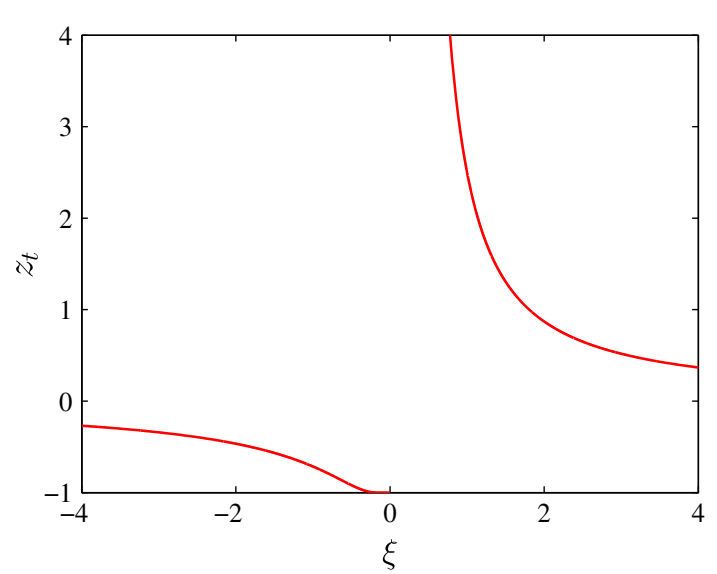

Fig. 2 Transition redshift $z_{t}$ at different parameter $\xi$ for the varying coupling model

\section{Observational data}

The observational constraints on the interacting dark energy models have been performed using the SNIa, BAO, OHD, $\mathrm{CMB}$. The first three observations mainly focus on the redshift range $0<z<2$. As a complement to previous work, we mainly forecast the ability of future SL tests into the deep redshift $2<z<5$. To track the evolution of the interaction over the redshift, we do not use all the observational data, but we only apply the most general SNIa and OHD at low redshift and the CMB for an early epoch as examples. However, we should note particularly that some priors on the parameters are needed in the calculation. Recently, two missions with high precision were released, i.e., WMAP-9 [36] and Planck 2013 [38]. For the Planck 2013 results, a low value of the Hubble constant $H_{0}$ and a high value of matter density parameter $\Omega_{m 0}$ are reported. This tension with other measurements immediately widely aroused people's concern. Therefore, in order to examine the effect of different priors on the parameters estimation, we will use the two sets of priors.

\subsection{SNIa}

The SNIa data are usually presented as the luminosity distance modulus. The updated available observation is from the Union2.1 compilation [39], which accommodates 580 data points. They are discovered by the Hubble Space Telescope Cluster Supernova Survey over the redshift interval $z<1.415$. Theoretically, the luminosity distance modulus is usually presented in the form of the difference between the apparent magnitude $m$ and the absolute magnitude $M$,

$\mu_{\mathrm{th}}(z)=m-M=5 \log _{10} D_{L}(z)+\mu_{0}$,

where $\mu_{0}=42.38-5 \log _{10} h$ and $h$ is the Hubble constant $H_{0}$ in units of $100 \mathrm{~km} \mathrm{~s}^{-1} \mathrm{Mpc}^{-1}$. The corresponding luminosity distance function $D_{L}(z)$ can be expressed as
$D_{L}(z)=(1+z) \int_{0}^{z} \frac{\mathrm{d} z^{\prime}}{E\left(z^{\prime} ; \mathbf{p}\right)}$,

where $\mathbf{p}$ stands for the parameter vector of each dark energy model embedded in the expansion rate parameter $E\left(z^{\prime} ; \mathbf{p}\right)$. Commonly, parameters in the expansion rate $E\left(z^{\prime} ; \mathbf{p}\right)$ including the annoying parameter $h$ can be determined by the general $\chi^{2}$ statistics. However, an alternative way is to marginalize over the "nuisance" parameter $\mu_{0}$ [40-42]. The rest of the parameters without $h$ can be estimated by minimizing

$\chi_{\mathrm{SN}}^{2}(z, \mathbf{p})=A-\frac{B^{2}}{C}$,

where

$$
\begin{aligned}
A(\mathbf{p}) & =\sum_{i} \frac{\left[\mu_{\mathrm{obs}}(z)-\mu_{\mathrm{th}}\left(z ; \mu_{0}=0, \mathbf{p}\right)\right]^{2}}{\sigma_{i}^{2}(z)}, \\
B(\mathbf{p}) & =\sum_{i} \frac{\mu_{\mathrm{obs}}(z)-\mu_{\mathrm{th}}\left(z ; \mu_{0}=0, \mathbf{p}\right)}{\sigma_{i}^{2}(z)}, \\
C & =\sum_{i} \frac{1}{\sigma_{i}^{2}(z)} .
\end{aligned}
$$

In fact, this program has been widely used in the cosmological constraints, such as the reconstruction of the dark energy [43], the parameter constraint [44], and the reconstruction of the energy condition history [45].

\section{$3.2 \mathrm{OHD}$}

The Hubble parameter $H(z)=\dot{a} / a$ is a key determining factor in the research of expansion history of the universe, because it has great relevance to various observations. In practice, we measure the Hubble parameter as a function of the redshift $z$. Observationally, we can deduce $H(z)$ from the differential ages of galaxies [46-48], from the BAO peaks in the galaxy power spectrum $[49,50]$ or from the BAO peak using the Ly $\alpha$ forest of QSOs [51]. In addition, we can also theoretically reconstruct $H(z)$ from the luminosity distances of SNIa using their differential relations [52-54]. Practically, the available OHD have been applied to constrain the standard cosmological model $[48,55]$ and some other FRW models [56-58]. Interestingly, the potential of future $H(z)$ observations in parameter constraint has also been explored [59]. In this paper, we use the latest available data listed in table 1 of Ref. [60], which accommodates 28 data points. The parameters can be estimated by minimizing

$\chi_{\mathrm{OHD}}^{2}(z, \mathbf{p})=\sum_{i} \frac{\left[H_{0} E\left(z_{i}\right)-H^{\mathrm{obs}}\left(z_{i}\right)\right]^{2}}{\sigma_{i}^{2}}$.

We have found that the nuisance parameter $H_{0}$ is embedded in (17). Therefore, in order to be immune from the influence of parameter $H_{0}$, we need to marginalize over it with a prior. In the calculation, we use the Gaussian prior 
$H_{0}=70.0 \pm 2.2 \mathrm{~km} \mathrm{~s}^{-1} \mathrm{Mpc}^{-1}$ suggested by WMAP-9 [36] and $H_{0}=67.3 \pm 1.2 \mathrm{~km} \mathrm{~s}^{-1} \mathrm{Mpc}^{-1}$ from Planck 2013 [38], respectively.

\section{$3.3 \mathrm{CMB}$}

The CMB experiment measures the temperature and polarization anisotropy of the cosmic radiation in the early epoch. It generally plays a major role in establishing and sharpening the cosmological models. The shift parameter $R$ is a convenient way to quickly evaluate the likelihood of the cosmological models. For the spatial flat model, it is expressed as

$$
R=\sqrt{\Omega_{m 0}} \int_{0}^{z_{s}} \frac{\mathrm{d} z^{\prime}}{E\left(z^{\prime} ; \mathbf{p}\right)},
$$

where $z_{s}=1090.97$ is the decoupling redshift $[36,61]$. According to the observation, we estimate the parameters by minimizing the corresponding $\chi^{2}$ statistics

$\chi_{R}^{2}=\left(\frac{R-1.728}{0.016}\right)^{2}$.

\subsection{Sandage-Loeb test}

The Sandage-Loeb (SL) test, i.e., the use of the redshift drift $\Delta z$, was first proposed by Sandage [26] in 1962. It is a very high potential measurement to directly probe the dynamics of cosmic expansion. In later decades, many observational candidates like masers and molecular absorptions lines were put forward [27]. At present, the E-ELT will be equipped with a high resolution, an extremely stable, ultra high precision spectrograph named the COsmic Dynamics EXperiment (CODEX), which is designed to be able to measure such signals in the near future. The scheduled E-ELT plans to measure the spectral shift of high-redshift QSOs [62]. These spectra are not only immune from the noise of the peculiar motions relative to the Hubble flow, but also they have a large number of lines in a single spectrum [63].

A signal emitted by a source at time $t_{\mathrm{em}}$ can be observed at time $t_{0}$. Because of the expansion of the universe, the source's redshift should be given through the scale factor

$z\left(t_{0}\right)=\frac{a\left(t_{0}\right)}{a\left(t_{\mathrm{em}}\right)}-1$.

Over the observer's time interval $\Delta t_{0}$, the source's redshift becomes

$z\left(t_{0}+\Delta t_{0}\right)=\frac{a\left(t_{0}+\Delta t_{0}\right)}{a\left(t_{\mathrm{em}}+\Delta t_{\mathrm{em}}\right)}-1$,

where $\Delta t_{\mathrm{em}}$ is the time interval-scale for the source to emit another signal. It should satisfy $\Delta t_{\mathrm{em}}=\Delta t_{0} /(1+z)$. The observed redshift change of the source is thus given by

$\Delta z=\frac{a\left(t_{0}+\Delta t_{0}\right)}{a\left(t_{\mathrm{em}}+\Delta t_{\mathrm{em}}\right)}-\frac{a\left(t_{0}\right)}{a\left(t_{\mathrm{em}}\right)}$.

A further relation can be obtained if we keep the first order approximation,

$\Delta z \approx\left[\frac{\dot{a}\left(t_{0}\right)-\dot{a}\left(t_{\mathrm{em}}\right)}{a\left(t_{\mathrm{em}}\right)}\right] \Delta t_{0}$.

Clearly, the observable $\Delta z$ is a direct change of the expansion rate during the evolution of the universe. In terms of the Hubble parameter $H(z)=\dot{a}\left(t_{\mathrm{em}}\right) / a\left(t_{\mathrm{em}}\right)$, it can be simplified as

$$
\frac{\Delta z}{\Delta t_{0}}=(1+z) H_{0}-H(z) \text {. }
$$

This is also known as the McVittie equation [64]. Taking a standard cosmological model as an example, we find that the redshift drift at low redshift generally appears to be negative with the predominance of the matter density parameter $\Omega_{m 0}$. This feature is often regarded as a method to distinguish dark energy models from LTB void models at $z<2$ (especially at low redshift) [65]. Unfortunately, the scheduled CODEX would not be able to measure the drift at such low $z$, since the target Ly $\alpha$ forest can be measured from the ground only at $z \geq$ 1.7 [28]. However, this expectation can be met by the precise HI $21 \mathrm{~cm}$ absorption line observations [66]. Observationally, it is more common to detect the spectroscopic velocity drift,

$\frac{\Delta v}{\Delta t_{0}}=\frac{c}{1+z} \frac{\Delta z}{\Delta t_{0}}$.

It can usually be detected at the order of several $\mathrm{cm} \mathrm{s}^{-1}$ year $^{-1}$. Obviously, the velocity variation $\Delta v$ is enhanced with the increase of the observational time $\Delta t_{0}$.

For the capability of CODEX, the accuracy of the spectroscopic velocity drift measurement was estimated by Pasquini et al. [63] using the Monte Carlo simulations

$\sigma_{\Delta v}=1.35\left(\frac{\mathrm{S} / \mathrm{N}}{2370}\right)^{-1}\left(\frac{N_{\mathrm{QSO}}}{30}\right)^{-1 / 2}\left(\frac{1+z_{\mathrm{QSO}}}{5}\right)^{q} \mathrm{~cm} / \mathrm{s}$,

with $q=-1.7$ for $2<z<4$, or $q=-0.9$ for $z>4$, where $\mathrm{S} / \mathrm{N}$ is the signal-to-noise ratio, $N_{\mathrm{QSO}}$ and $z_{\mathrm{QSO}}$ are, respectively, the number and redshift of the observed QSO. According to currently known QSOs brighter than 16.5 in $2<z<5$, we adopt the assumption in Refs. [63] with $N_{\mathrm{QSO}}=30$ and a $\mathrm{S} / \mathrm{N}$ of 3000 . Using the simulations, the SL test has been widely applied in the model constraints $[31,34,67,68]$, assumed to be uniformly distributed among specific redshift bins. Following previous work, we would like to examine the ability of the SL test on the interacting dark energy models of concern. The mock $\Delta v$ data for 10 years are assumed to obey a uniform distribution among

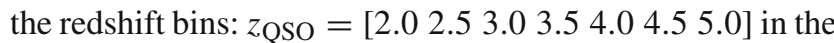



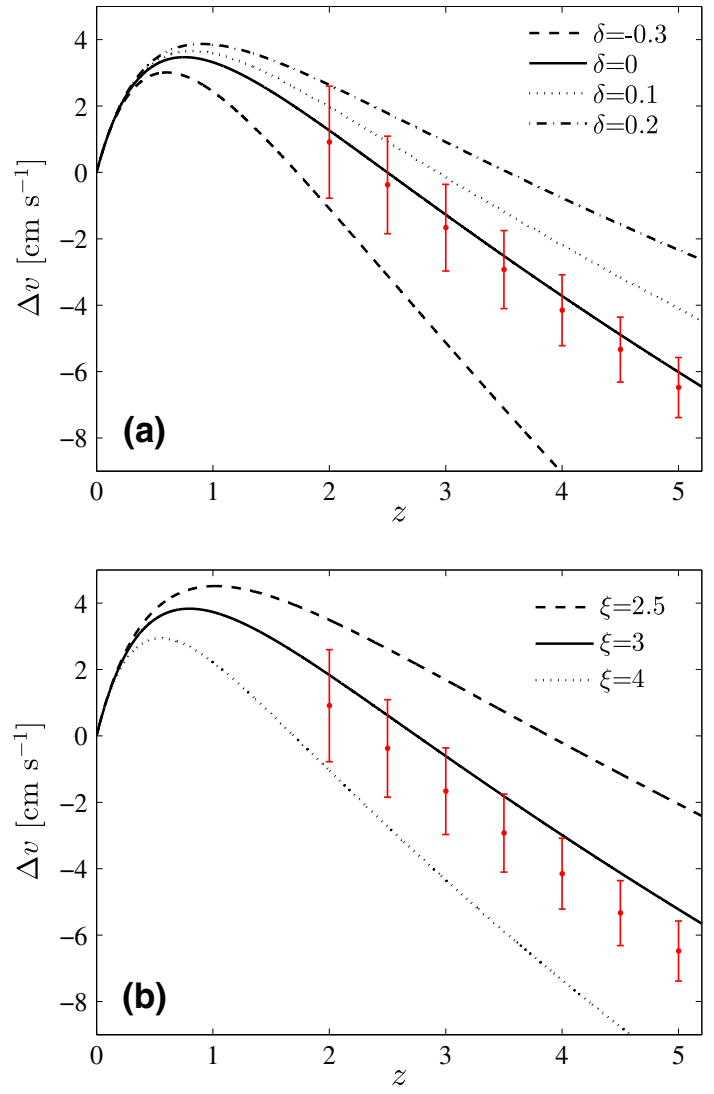

Fig. 3 Comparison between the simulated $\Delta v$ over 10 year observational time and theoretical expectations of the evaluated a constant coupling model and $\mathbf{b}$ varying coupling model for different parameters. For model a, we change the interaction term $\delta$ and fix the other parameters under the best estimation by Guo et al. [22]. For model b, we change the parameter $\xi$ and fix the other parameters as the best estimation by Cao et al. [24]. The simulated data points with error bars are estimated by (26) in the fiducial model

fiducial concordance cosmological model. Their errors can be calculated from the estimation of (26). Similar to the $\chi^{2}$ statistics of OHD, we should use some priors in the calculation. As introduced above, we use two sets of priors. During the simulation, the parameters of the fiducial $\Lambda \mathrm{CDM}$ model are given by the best-fit values of WMAP-9 [36] and Planck 2013 [38]. For example, the matter density parameter suggested by the WMAP- 9 measurement is $\Omega_{m 0}=0.279$, while Planck 2013 reports a higher value, i.e., $\Omega_{m 0}=0.315$.

In Fig. 3, we plot the predicted $\Delta v$ for different models with different parameters. We find that the predicted $\Delta v$ curves extend away from each other at high redshift. In fact, it is useful to precisely determine the parameters. Comparing with the simulated $\Delta v$, we find that the parameters are constrained in the narrow regions. For example in the constant coupling model, if we fix $w_{X}$ and $\Omega_{X 0}$ as the best estimation by Guo et al. [22], we find that the interaction term $\delta \sim[-0.3,0.2]$ seems to be favored as shown in panel (a). For the varying coupling model, the parameter $\xi \sim[2.5,4]$ seems to be favored when we fix the other parameters as the best estimation by Cao et al. [24]. Nevertheless, a precise determination of the parameters should minimize the corresponding $\chi^{2}$ statistics,

$\chi_{\Delta v}^{2}(z, \mathbf{p})=\sum_{i} \frac{\left[\Delta v^{\text {model }}\left(z_{i}\right)-\Delta v^{\text {data }}\left(z_{i}\right)\right]^{2}}{\sigma_{\Delta v}^{2}\left(z_{i}\right)}$,

where $\Delta v^{\text {model }}\left(z_{i}\right)$ is the theoretical expectation of the evaluated dark energy models, i.e., the constant and varying coupling models. $\Delta v^{\text {data }}\left(z_{i}\right)$ is for the mock data produced in the fiducial $\Lambda \mathrm{CDM}$ model, and $\sigma_{\Delta v}^{2}\left(z_{i}\right)$ is the corresponding error estimated by (26).

In general, we often perform a joint analysis by combining several types of observational data in order to better constrain the cosmological models. In this paper, we will, respectively, perform the likelihood fit by adding different types of observational data in order to test the evolution of the interaction term.

\section{Constraint on the coupling models}

By performing the $\chi^{2}$-test using different datasets, we are able to give the constraint on the parameters, and we reconstruct the evolution of the interaction term. Because we have used two sets of parameter priors in the calculation, we would like to report the constraint results, respectively.

\subsection{WMAP-9 priors}

For the constant coupling model, we implement the likelihood analysis using different datasets and display the corresponding contour constraints of parameters $\left(w_{X}, \delta\right)$ in Figs. 4 and 5, after marginalizing over the current dark energy density parameter $\Omega_{X 0}$. For the observational data combination $\mathrm{SNIa}+\mathrm{OHD}+\mathrm{CMB}$, they give a very severe constraint on the interaction term, $\delta=-0.01 \pm 0.02(1 \sigma) \pm 0.04(2 \sigma)$, which represents a weak but negative interaction between dark energy and matter. The rest of the EoS parameters and the dark energy density are also constrained to a good level, $w_{X}=-1.015_{-0.17}^{+0.14}(2 \sigma)$ and $\Omega_{X 0}=0.72_{-0.04}^{+0.04}(2 \sigma)$. In order to forecast the ability of the SL test, we add the simulated SL test data with current data, and we show the results in the middle panel of Fig. 4. We find that the SL test effectively reduces the contour constraint region. Especially, the key parameter interaction term is more stringently constrained, which can be seen from the marginalized probability distribution (PDF) of $\delta$ in the bottom panel. The corresponding constraints are $\delta=-0.01 \pm 0.01(1 \sigma) \pm 0.02(2 \sigma), w_{X}=-1.01_{-0.11}^{+0.10}(2 \sigma)$, and $\Omega_{X 0}=0.72_{-0.01}^{+0.02}(2 \sigma)$, respectively. Previous work found that the observational data apart from the CMB cannot constrain the interacting models well. We perform the same 

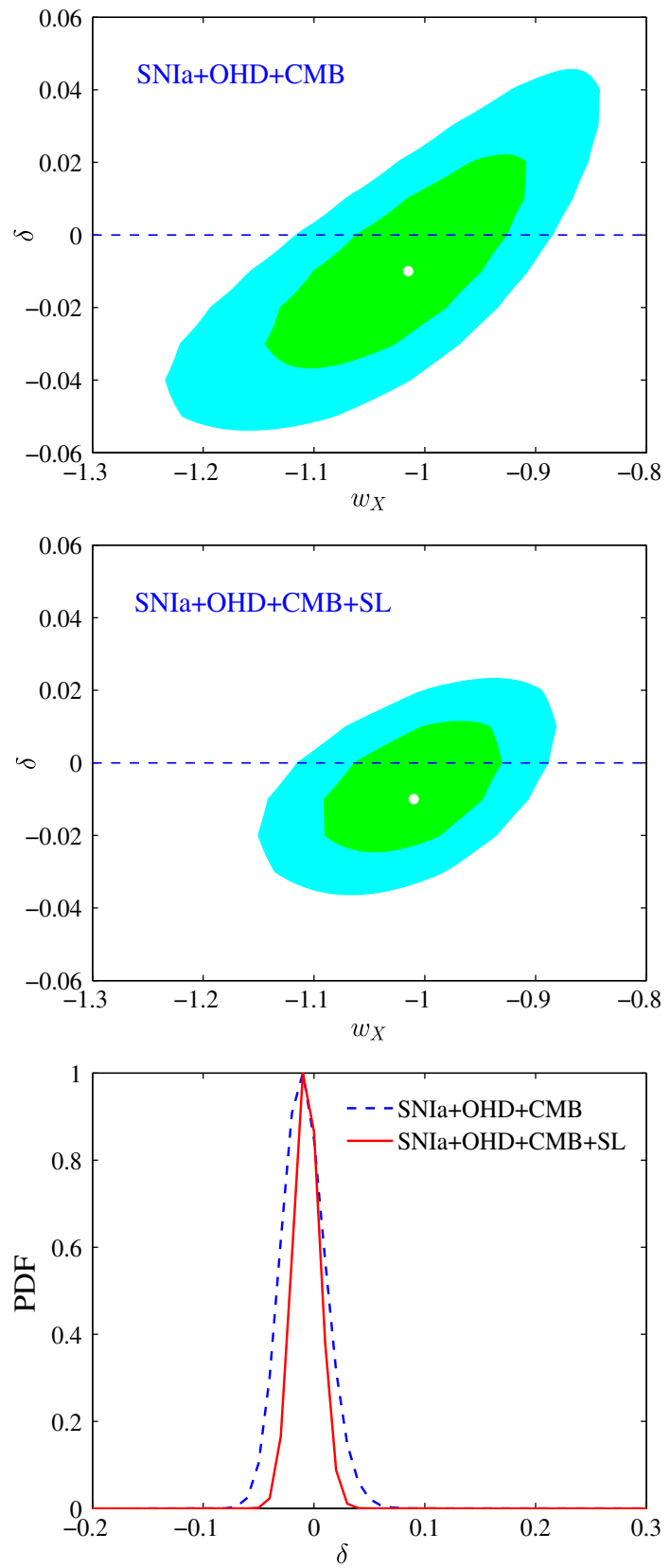

Fig. 4 Contours correspond to $68.3 \%, 95.4 \%$ confidence levels and the marginalized probability distribution of $\delta$ with different datasets for the constant coupling model. The prior is from WMAP-9 [36]

likelihood test from the joint analysis of SNIa and OHD and obtain $\delta=0.39_{-0.90}^{+0.40}(2 \sigma)$, which is much more rough compared with the inclusion of CMB. As stated by Guo et al. [22], this is because a large coupling can change the cosmological evolution during the matter-dominated epoch. In order to further track the evolution of the interaction with the expansion of the universe, we extend our analysis to the higher redshift using the SL test in Fig. 5. We find that the inclusion of the SL test much improves the constraint, $\delta=0.10_{-0.36}^{+0.25}(2 \sigma)$. The contour constraint region at $2 \sigma$ level with the SL test
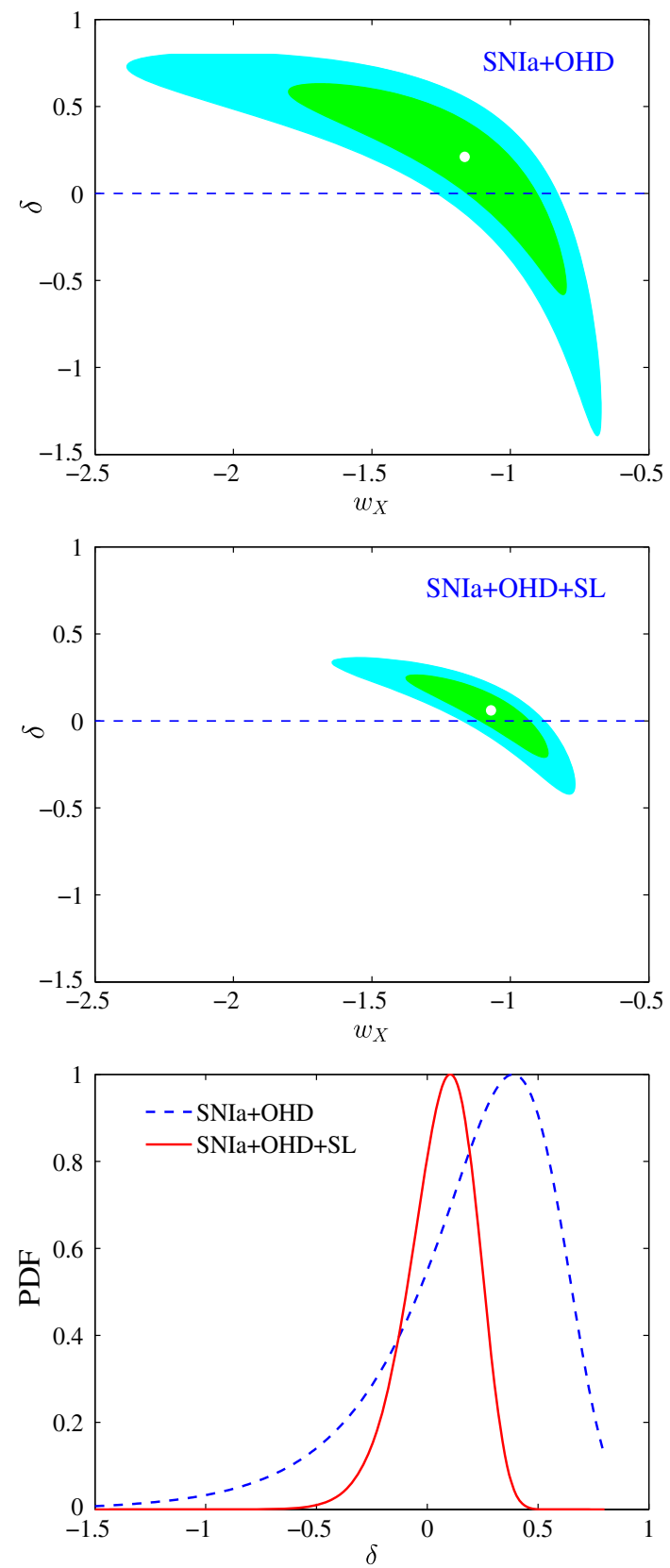

Fig. 5 Same as Fig. 4 but for different datasets

is even smaller than the constraint without SL at $1 \sigma$ level. The marginalized PDF of the interaction $\delta$ is not only narrowed with a high significance, but it also moves towards zero.

For the varying coupling model, we perform the same likelihood test using the current observational data with or without SL test, respectively. For the combination of all considered current observational data, we find that they can provide fair constraints on the parameters. For example, the EoS and dark energy density parameters are $w_{X}=-1.02_{-0.16}^{+0.14}$ $(2 \sigma), \Omega_{X 0}=0.72_{-0.04}^{+0.04}(2 \sigma)$, respectively. The parameter 

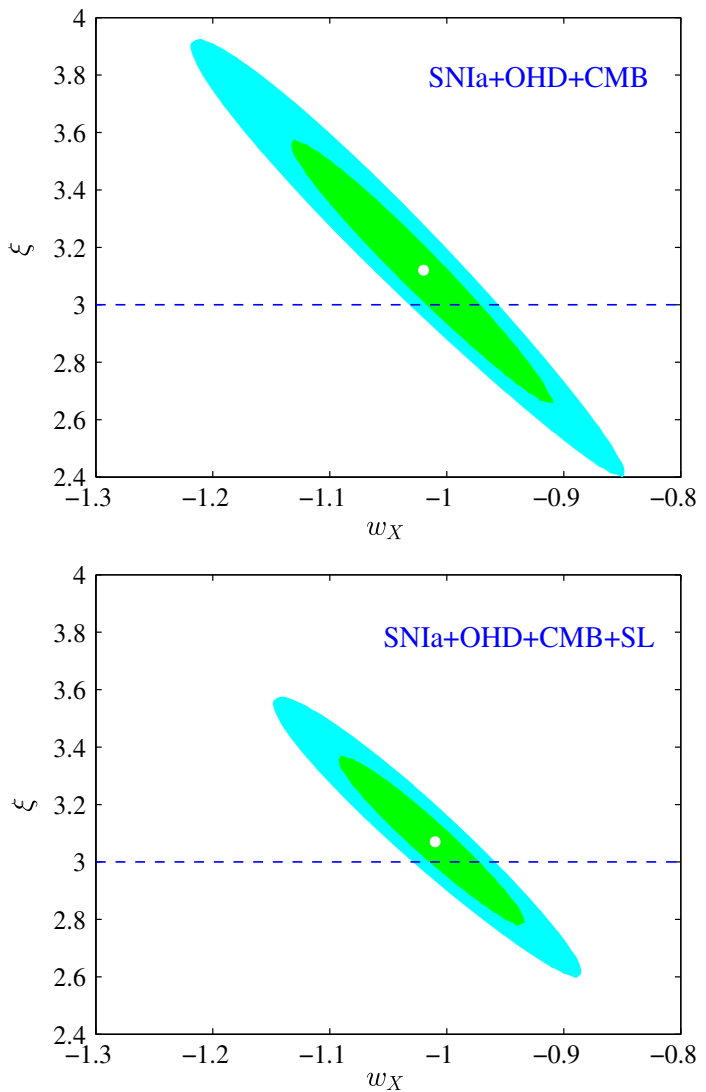

Fig. 6 Contours correspond to $68.3 \%, 95.4 \%$ confidence levels for the varying coupling model with different datasets. The prior is from WMAP-9 [36]

$\xi=3.12_{-0.29}^{+0.31}(1 \sigma)_{-0.57}^{+0.66}(2 \sigma)$ shows that the coincidence problem is still severe. From (10), we find that the sign of the interaction term completely depends on the current value $\delta_{0}=-\left(\xi+3 w_{X}\right) \Omega_{X 0}$. Using the constraint results, we reconstruct the current interaction, $\delta_{0}=-0.04_{-0.28}^{+0.26}(1 \sigma)$, which shows that the interaction today is weak but has a negative best-fit value from the available observations. This is consistent with previous results [22]. For forecasting the power of the SL test, we also include the mock SL data in the bottom panel of Fig. 6 . The allowed region of the parameters is obviously reduced, which is the same as in the constant coupling model. The parameters in this case are found to be $w_{X}=-1.01_{-0.11}^{+0.10}(2 \sigma), \Omega_{X 0}=0.725_{-0.02}^{+0.02}(2 \sigma)$, and $\xi=3.07_{-0.19}^{+0.20}(1 \sigma)_{-0.37}^{+0.41}(2 \sigma)$. Moreover, the phantom-like dark energy $\left(w_{X}<-1\right)$ is slightly favored over the $\Lambda \mathrm{CDM}$ model. The interaction is thus reconstructed in Fig. 10 with a current value $\delta_{0}=-0.02_{-0.19}^{+0.17}(1 \sigma)$. We find that the bestfit interaction is negative but with relatively large errors for the low redshift, which is a hint that we have energy transfer from matter to dark energy. We also note that the interaction $\delta(z)$ decreases with the increase of redshift $z$ and approaches zero at infinity.
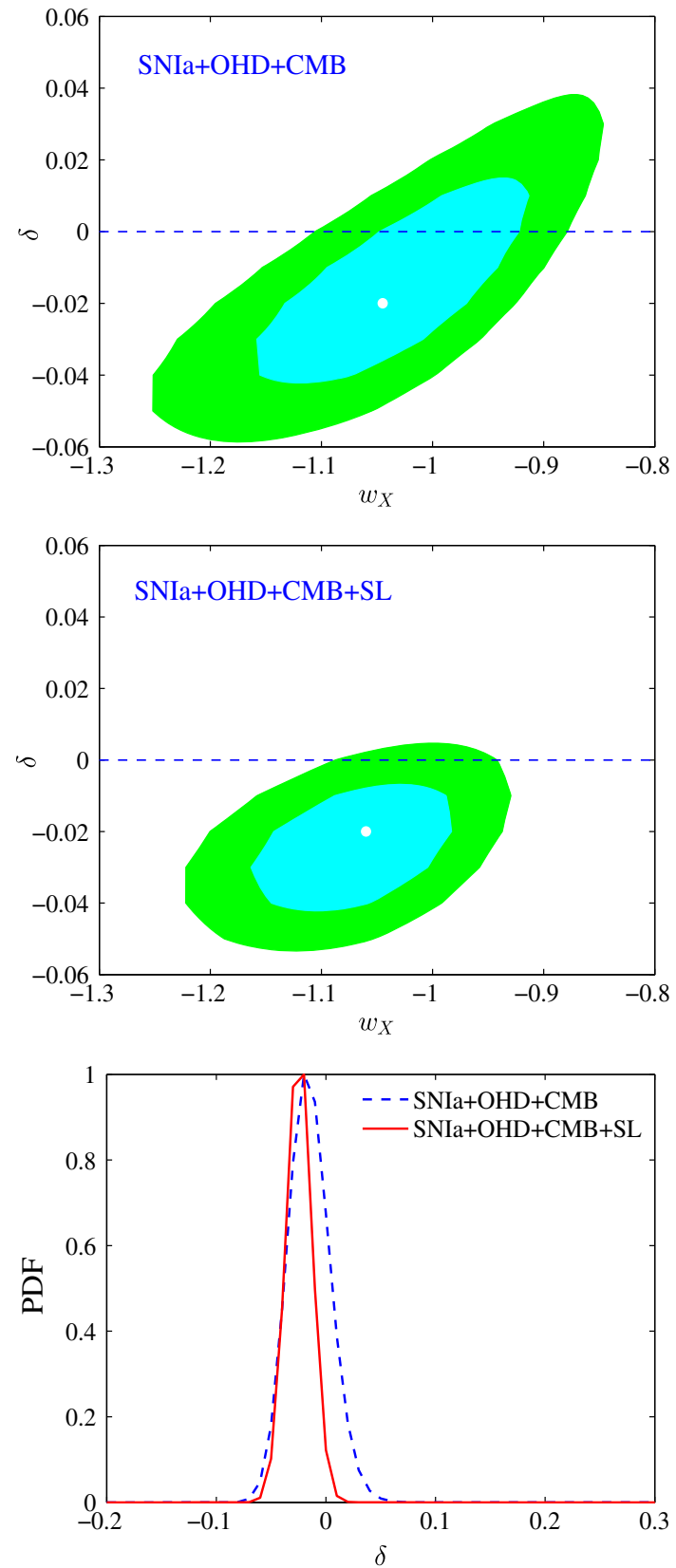

Fig. 7 Contours correspond to $68.3 \%, 95.4 \%$ confidence levels and the marginalized probability distribution of $\delta$ with different datasets for the constant coupling model. The prior is from Planck 2013 [38]

\subsection{Planck priors}

Under the Planck priors, we plot the results of the constant coupling model in Figs. 7 and 8. The available dataset SNIa+OHD+CMB shows that the interaction term is $\delta=$ $-0.02_{-0.01}^{+0.02}(1 \sigma)_{-0.03}^{+0.04}(2 \sigma)$. Although the central value changes slightly to higher values, this estimation is consistent with the result in WMAP-9 prior, i.e., $-0.05<\delta<0.03$ within $2 \sigma$ C.L. The rest of the parameters, $w_{X}=-1.03_{-0.17}^{+0.15}$ $(2 \sigma)$ and $\Omega_{X 0}=0.71_{-0.04}^{+0.04}(2 \sigma)$, also agree with the results 

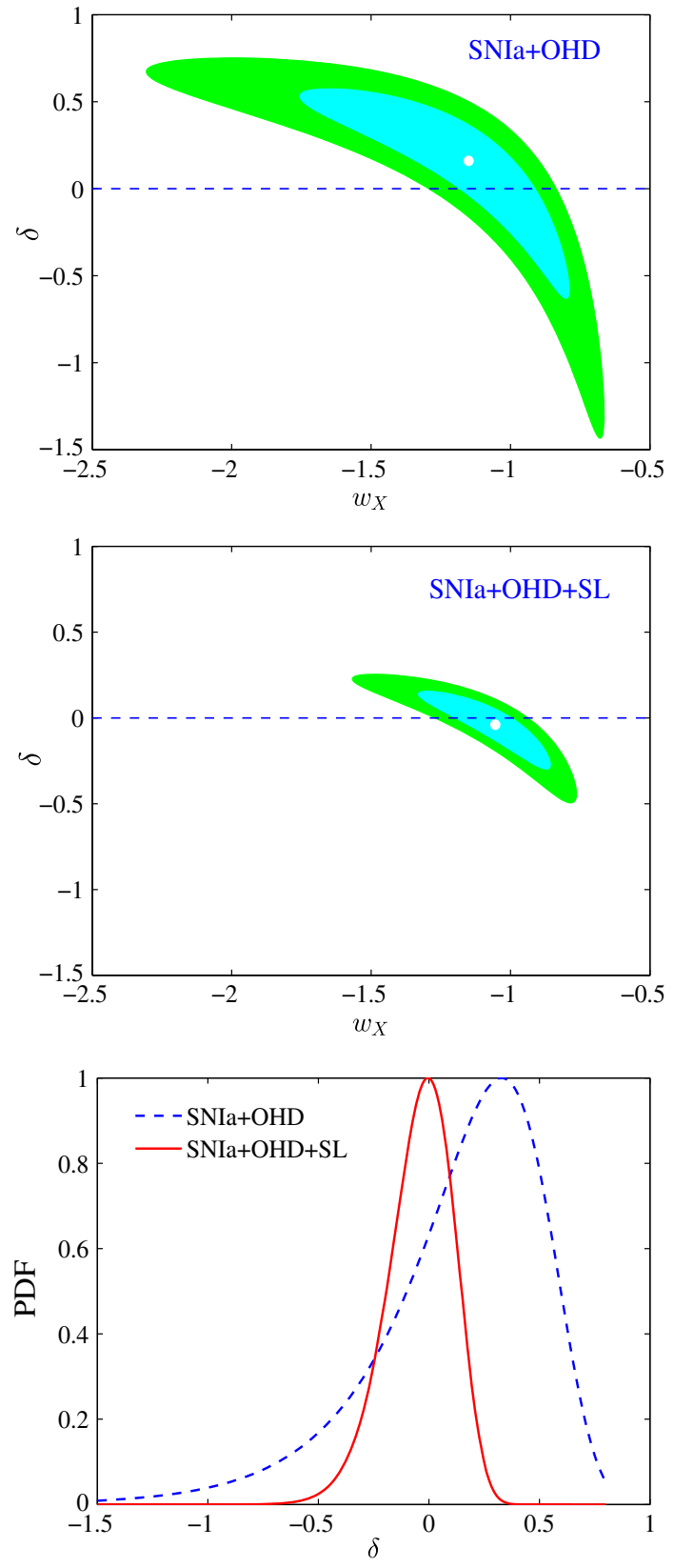

Fig. 8 Same as Fig. 7, but for different datasets

in WMAP-9 prior. With the inclusion of the SL test, we find that the constraint on the interaction term is $\delta=-0.02_{-0.016}^{+0.007}$ $(1 \sigma)_{-0.03}^{+0.02}(2 \sigma)$. We should note that the interaction in this scenario is negative at high confidence level, i.e., $-0.05<$ $\delta<0$, which is different from the result of WMAP-9, $-0.03<\delta<0.02(2 \sigma)$. That is, the Planck prior favors an energy transfer from matter to dark energy with high significance. As described in above subsection, Guo et al. [22] found that a large interaction is not allowed at high redshift. In order to track the key redshift spot, we have to revisit the constraint of observations at low redshift. We find that a combination of SNIa and OHD in the Planck priors gives
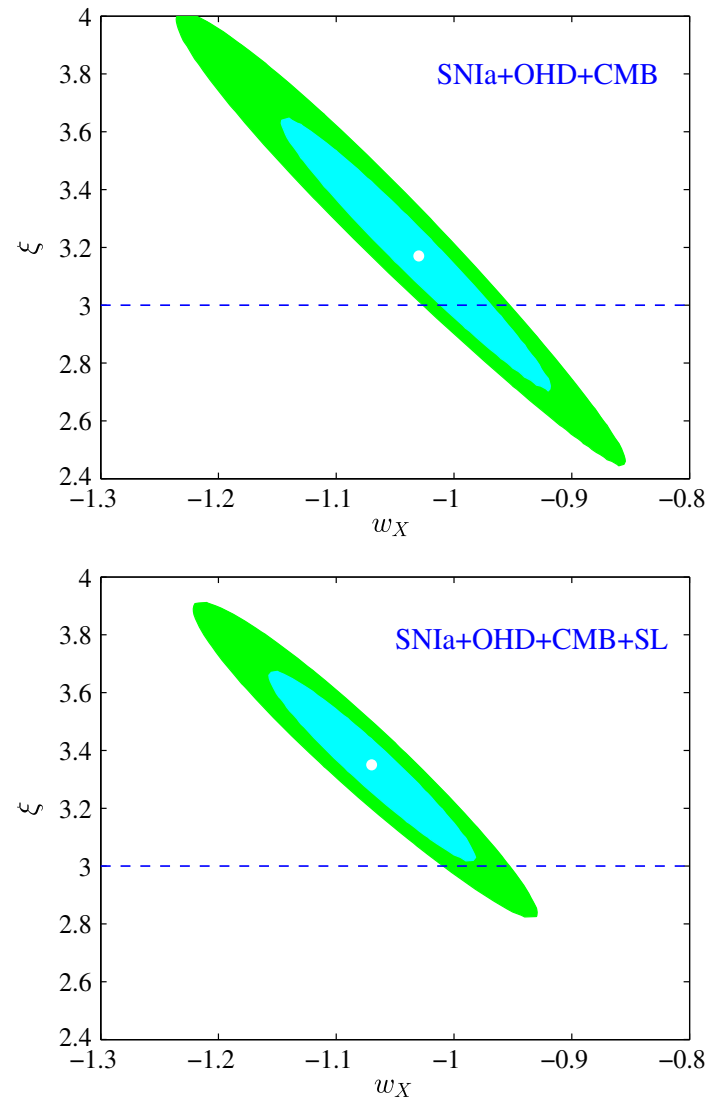

Fig. 9 Contours correspond to $68.3 \%, 95.4 \%$ confidence levels for the varying coupling model with different datasets. The prior is from Planck 2013 [38]

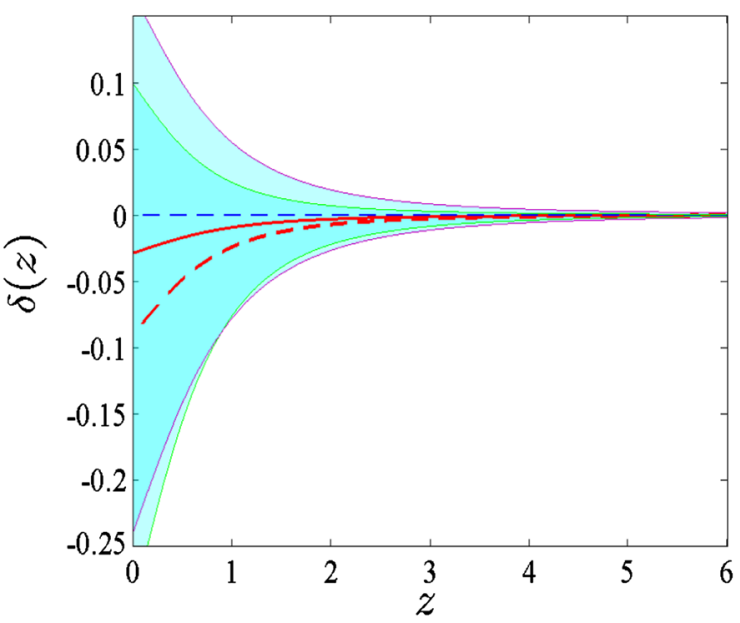

Fig. 10 Reconstruction of the interaction term $\delta(z)$ using the total considered data for the varying coupling model. The shaded region corresponds to the errors of $\delta(z)$ at $1 \sigma$ C.L. The central solid and dashed curves are the best-fit $\delta(z)$. The light-colored region and red solid curve correspond to the reconstruction in WMAP-9 prior; the thick-colored region and the red dashed curve correspond to the reconstruction in the Planck 2013 prior 
$\delta=0.33_{-0.34}^{+0.23}(1 \sigma)_{-0.91}^{+0.41}(2 \sigma)$, a similar estimation to the result in WMAP-9 priors. However, this result immediately changes with the inclusion of SL test. The combination SNIa, OHD, and SL leads to $\delta=0_{-0.35}^{+0.24}(2 \sigma)$. Firstly, we once again find evidence for the power of the SL test in this scenario, i.e., the contour constraint region at $2 \sigma$ level with the SL test is comparable with the constraint without SL at $1 \sigma$ level, which is the same as the situation in WMAP-9 priors. Secondly, the central value of the interaction term directly changes from 0.33 to 0 , which is shown in the bottom panel of Fig. 8. Comparing the PDF of Figs. 5 and 8, we find that the Planck 2013 priors move the constraint towards the direction of non-interaction, faster than the constraint in WMAP-9 priors.

For the varying coupling model, we also perform the related analysis in the Planck 2013 priors and plot the constraints in Fig. 9. The available data combination shows $\xi=3.18_{-0.30}^{+0.33}(1 \sigma)$. Similar to the constant coupling model, the new priors make the results from current observational data change slightly, which can be witnessed from the comparison between the top panel of Figs. 6 and 9. However, we note that the Planck priors promote an important change on the SL test. By including the SL test with current observational data, they give a bigger estimation on the key parameter, $\xi=3.34_{-0.21}^{+0.23}(1 \sigma)_{-0.41}^{+0.47}(2 \sigma)$. Firstly, we should note that the errors of $\xi$ are significantly reduced with the inclusion of the SL test. Secondly, it is very important that the parameter $\xi>3$ at $1 \sigma$ C.L. As stated above, the constant $\xi$ characterizes the severity of the coincidence problem. If $\xi=3$, this model can recover the $\Lambda \mathrm{CDM}$ model, and the coincidence problem is still alive. Obviously, $\xi>3$ at $1 \sigma$ C.L. indicates that the coincidence problem will be less severe. Moreover, the EoS of dark energy $w_{X}=-1.07_{-0.06}^{+0.06}(1 \sigma)$, which indicates that the combination including the SL test in this prior remarkably favors a phantom-like dark energy. The terms $\xi+3 w_{X}=0$ and $\xi+3 w_{X} \neq 0$, respectively, denote the standard cosmology without interaction and nonstandard cosmology. The reconstruction from data including the SL test is $\xi+3 w_{X}=0.13_{-0.27}^{+0.29}(1 \sigma)$. The interaction term today is $\delta_{0}=-0.09_{-0.20}^{+0.19}(1 \sigma)$, which is slightly stronger than the result $\delta_{0}=-0.02$ in WMAP-9 priors. We then reconstruct the $\delta(z)$ and compare it with the reconstruction under WMAP-9 priors in Fig. 10. We find that they behave in the same way for a high redshift. For a low redshift, the interaction in the Planck priors is slightly stronger. Moreover, the reconstruction with errors at $1 \sigma$ C.L. prefers $\delta<0$, i.e., an energy transfer from matter to dark energy.

\section{Conclusion and discussion}

The constant $\delta[18,19]$ and varying coupling $\delta(z)$ dark energy models [15] have been revisited using the secular Sandage-
Loeb (SL) test. The SL test is in the inaccessible redshift zone for recent observations, such as the SNIa, OHD, and BAO at $z<2$ and the CMB at $z \simeq 1090$. We have extended the analysis to the epoch at $2<z<5$ using the secular redshift drift of the QSO spectra. For convenience of the calculation, we use different priors from the WMAP-9 [36] and Planck 2013 [38], respectively.

For the constant coupling model, the current observation combinations in the two sets of priors both give a weak interaction term, which is consistent with previous results [22]. By including the simulated SL test data, we find that they can more stringently constrain the corresponding parameters; the interaction in the WMAP-9 priors is $\delta=-0.01 \pm 0.01$ $(1 \sigma) \pm 0.02(2 \sigma)$, which has been improved to be only half of the original scale on the errors. We should also note that the interaction in the Planck priors is negative at $2 \sigma$ C.L., which favors an energy transfer from matter to dark energy. As stated by Guo et al. [22], a large interaction is not allowed during the standard matter-dominated epoch; otherwise it can modify the CMB angular-diameter distance. We, therefore, analyze the interaction from late time observations. We extend the analysis to the redshift interval $2<z<5$ and compare it with a combination of SNIa and OHD. We find that the SL test can much more stringently constrain the parameters. The best fit of the interaction term in the Planck prior is directly reduced from 0.33 to zero. Therefore, the higherredshift observation including the SL test is necessary for revealing how the interaction gradually changes with the cosmic evolution.

For the varying coupling model, the relation $\rho_{X} \propto \rho_{m} a^{\xi}$ is imposed on the density evolution of the cosmic components. Combining the SL test with the current observational data, we find that they can present a more narrowed constraint, which behaves similar to the constant coupling model. In this scenario, the key parameter $\xi$ characterizes the severity of the coincidence problem. The $\Lambda \mathrm{CDM}$ model can be reduced for the case $\xi=3$. The terms $\xi+3 w_{X}=0$ and $\xi+3 w_{X} \neq 0$, respectively, denote the standard cosmology without interaction and non-standard cosmology. From the likelihood test in Planck 2013 priors, we find the term $\xi+3 w_{X}=0.13_{-0.27}^{+0.29}$ $(1 \sigma)$. Importantly, the parameter $\xi$ at $1 \sigma$ C.L. in Planck priors is $\xi>3$, which indicates that the coincidence problem will be less severe. We also reconstruct the interaction $\delta(z)$ in Fig. 10. It is found that the best-fit $\delta(z)$ is negative at low redshift and generally vanishes for high redshift. Moreover, the errors of the reconstructed $\delta(z)$ are remarkable at low redshift. However, the phantom-like dark energy with $w_{X}<-1$ is favored over the $\Lambda \mathrm{CDM}$ model.

An investigation using the SL test on the constant coupling model shows that the interaction with errors up to redshift $z \sim 5$ cannot be neglected. Therefore, it is also reasonable for us to deduce that the observations at higher redshift, such as the gamma-ray burst may be useful to detect the interacting 
model, because some of them even can be monitored at the redshift $z \sim 8$. We should also note that the inclusion of the SL test with a small sample can significantly narrow the contour region from the large sample data, which can be found evidence for in Figs. 5 and 8. Furthermore, the SL test is immune against the model-dependence, calibration of the standard candle, and the peculiar motion of the observed objects. Therefore, we could expect that future SL tests will play an important role to constrain the cosmological models.

Acknowledgments We quite appreciate the anonymous referee for his/her suggestions to improve this manuscript. M.J. Zhang would like to thank Zhengxiang Li and Jing-Zhao Qi for their valuable and kind help on programming. This work was supported by the National Natural Science Foundation of China (Grant Nos. 11235003, 11175019, 11178007).

Open Access This article is distributed under the terms of the Creative Commons Attribution License which permits any use, distribution, and reproduction in any medium, provided the original author(s) and the source are credited.

Funded by $\mathrm{SCOAP}^{3}$ / License Version CC BY 4.0.

\section{References}

1. A.G. Riess, A.V. Filippenko, P. Challis, A. Clocchiatti, A. Diercks, P.M. Garnavich, R.L. Gilliland, C.J. Hogan, S. Jha, R.P. Kirshner et al., Astronom. J. 116(3), 1009 (2007)

2. M. Tegmark, M.A. Strauss, M.R. Blanton, K. Abazajian, S. Dodelson, H. Sandvik, X. Wang, D.H. Weinberg, I. Zehavi, N.A. Bahcall et al., Phys. Rev. D 69(10), 103501 (2004)

3. D.N. Spergel, L. Verde, H.V. Peiris, E. Komatsu, M. Nolta, C. Bennett, M. Halpern, G. Hinshaw, N. Jarosik, A. Kogut et al., Astrophys. J. Suppl. Ser. 148(1), 175 (2003)

4. S. Weinberg, Rev. Mod. Phys. 61(1),1 (1989)

5. S. Weinberg, arXiv preprint astro-ph/0005265 (2000)

6. I. Zlatev, L. Wang, P.J. Steinhardt, Phys. Rev. Lett. 82(5), 896 (1999)

7. A. Vilenkin, The Dark Universe: Matter, Energy and Gravity, vol. 15 (2003), p. 173

8. J. Garriga, M. Livio, A. Vilenkin, Phys. Rev. D 61(2), 023503 (1999)

9. J. Garriga, A. Vilenkin, Phys. Rev. D 64(2), 023517 (2001)

10. E.J. Copeland, M. Sami, S. Tsujikawa, Int. J. Mod. Phys. D 15(11), 1753 (2006)

11. B. Ratra, P.J. Peebles, Phys. Rev. D 37(12), 3406 (1988)

12. R.R. Caldwell, Phys. Lett. B 545(1), 23 (2002)

13. C. Armendariz-Picon, V. Mukhanov, P.J. Steinhardt, Phys. Rev. D 63(10), 103510 (2001)

14. B. Feng, X. Wang, X. Zhang, Phys. Lett. B 607(1), 35 (2005)

15. N. Dalal, K. Abazajian, E. Jenkins, A.V. Manohar, Phys. Rev. Lett. 87(14), 141302 (2001)

16. D. Behnke, D.B. Blaschke, V.N. Pervushin, D. Proskurin, Phys. Lett. B 530(1), 20 (2002)

17. S.M. Carroll, L. Mersini, Phys. Rev. D 64(12), 124008 (2001)

18. P. Wang, X.H. Meng, Class. Quantum Gravity 22(2), 283 (2005)

19. L. Amendola, G.C. Campos, R. Rosenfeld, Phys. Rev. D 75(8), 083506 (2007)

20. H. Wei, Nucl. Phys. B 845(3), 381 (2011)

21. F. Costa, J. Alcaniz, Phys. Rev. D 81(4), 043506 (2010)
22. Z.K. Guo, N. Ohta, S. Tsujikawa, Phys. Rev. D 76(2), 023508 (2007)

23. Y. Chen, Z.H. Zhu, J. Alcaniz, Y. Gong, Astrophys. J. 711(1), 439 (2010)

24. S. Cao, N. Liang, Z.H. Zhu, Mon. Not. R. Astron. Soc. 416(2), $1099(2011)$

25. V. Salvatelli, A. Marchini, L. Lopez-Honorez, O. Mena, Phys. Rev. D 88(2), 023531 (2013)

26. A. Sandage, Astrophys. J. 136, 319 (1962)

27. A. Loeb, Astrophys. J. Lett. 499(2), L111 (1998)

28. J. Liske, A. Grazian, E. Vanzella, M. Dessauges, M. Viel, L. Pasquini, M. Haehnelt, S. Cristiani, F. Pepe, G. Avila et al., Mon. Not. R. Astron. Soc. 386(3), 1192 (2008)

29. J. Liske, A. Grazian, E. Vanzella, M. Dessauges, M. Viel, L. Pasquini, M. Haehnelt, S. Cristiani, F. Pepe, P. Bonifacio et al., Messenger 133, 10 (2008)

30. J. Liske, L. Pasquini, P. Bonifacio, F. Bouchy, R. Carswell, S. Cristiani, M. Dessauges, S. D’Odorico, V. D'Odorico, A. Grazian et al., Science with the VLT in the ELT Era (Springer, Netherlands, 2009), pp. 243-247. doi:10.1007/978-1-4020-9190-2_41

31. H. Zhang, W. Zhong, Z.H. Zhu, S. He, Phys. Rev. D 76(12), 123508 (2007)

32. D. Jain, S. Jhingan, Phys. Lett. B 692(4), 219 (2010)

33. J. Zhang, L. Zhang, X. Zhang, Phys. Lett. B 691(1), 11 (2010)

34. Z. Li, K. Liao, P. Wu, H. Yu, Z.H. Zhu, Phys. Rev. D 88(2), 023003 (2013)

35. B. Moraes, D. Polarski, Phys. Rev. D 84(10), 104003 (2011)

36. G. Hinshaw, D. Larson, E. Komatsu, D. Spergel, et al., Astrophys. J. 208(2), 19 (2013)

37. A.G. Riess, L.G. Strolger, J. Tonry, S. Casertano, H.C. Ferguson, B. Mobasher, P. Challis, A.V. Filippenko, S. Jha, W. Li et al., Astrophys. J. 607(2), 665 (2004)

38. Planck Collaboration, P.A.R. Ade, N. Aghanim, C. ArmitageCaplan, M. Arnaud, M. Ashdown, F. Atrio-Barandela, J. Aumont, C. Baccigalupi, A.J. Banday et al., ArXiv e-prints: 1303.5076 (2013)

39. N. Suzuki, D. Rubin, C. Lidman, G. Aldering, R. Amanullah, K. Barbary, L. Barrientos, J. Botyanszki, M. Brodwin, N. Connolly et al., Astrophys. J. 746(1), 85 (2012)

40. E.D. Pietro, J.F. Claeskens, Mon. Not. R. Astron. Soc. 341(4), 1299 (2003)

41. S. Nesseris, L. Perivolaropoulos, Phys. Rev. D 72(12), 123519 (2005)

42. L. Perivolaropoulos, Phys. Rev. D 71(6), 063503 (2005)

43. H. Wei, N. Tang, S.N. Zhang, Phys. Rev. D 75(4), 043009 (2007)

44. H. Wei, J. Cosmol. Astropart. Phys. 2010(08), 020 (2010)

45. C.J. Wu, C. Ma, T.J. Zhang, Astrophys. J. 753(2), 97 (2012)

46. R. Jimenez, A. Loeb, Astrophys. J. 573(1), 37 (2008)

47. J. Simon, L. Verde, R. Jimenez, Phys. Rev. D 71(12), 123001 (2005)

48. D. Stern, R. Jimenez, L. Verde, M. Kamionkowski, S.A. Stanford, J. Cosmol. Astropart. Phys. 2010(02), 008 (2010)

49. E. Gaztanaga, A. Cabre, L. Hui, Mon. Not. R. Astron. Soc. 399(3), $1663(2009)$

50. M. Moresco, A. Cimatti, R. Jimenez, L. Pozzetti, G. Zamorani, M. Bolzonella, J. Dunlop, F. Lamareille, M. Mignoli, H. Pearce et al., J. Cosmol. Astropart. Phys. 2012(08), 006 (2012)

51. N.G. Busca, T. Delubac, J. Rich et al., Astron. Astrophys. 552, A96 (2013). doi:10.1051/0004-6361/201220724

52. Y. Wang, M. Tegmark, Phys. Rev. D 71(10), 103513 (2005)

53. A. Shafieloo, U. Alam, V. Sahni, A.A. Starobinsky, Mon. Not. R. Astron. Soc. 366(3), 1081 (2006)

54. C. Mignone, M. Bartelmann, Astron. Astrophys. 481(2), 295 (2008)

55. H. Lin, H. Cheng, X. Wang, Y. Qiang, Y. Ze-Long, T.J. Zhang, B.Q. Wang, Mod. Phys. Lett. A 24(21), 1699 (2009)

56. L. Samushia, B. Ratra, Astrophys. J. Lett. 650(1), L5 (2008) 
57. T.J. Zhang, C. Ma, T. Lan, Adv. Astron. 2010, 81 (2010)

58. Z.X. Zhai, T.J. Zhang, W.B. Liu, J. Cosmol. Astropart. Phys. 2011(08), 019 (2011)

59. C. Ma, T.J. Zhang, Astrophys. J. 730(2), 74 (2011)

60. O. Farooq, B. Ratra, Astrophys. J. Lett. 766(1), L7 (2013)

61. W. Hu, N. Sugiyama, Astrophys. J. 471(2), 542 (1996)

62. L. Pasquini, S. Cristiani, H. Dekker, et al., Proceedings of the International Astronomical Union, vol. 1 (Cambridge University Press, 2006), p. 193

63. L. Pasquini, S. Cristiani, H. Dekker, M. Haehnelt, P. Molaro, F. Pepe, G. Avila, B. Delabre, S. D'Odorico, J. Liske et al., Messenger 122, 10 (2005)
64. G. McVittie, Astrophys. J. 136, 334 (1962)

65. C.M. Yoo, T. Kai, K.I. Nakao, Phys. Rev. D 83(4), 043527 (2011)

66. J. Darling, Astrophys. J. Lett. 761(2), L26 (2012)

67. A. Balbi, C. Quercellini, Mon. Not. R. Astron. Soc. 382(4), 1623 (2007)

68. P.S. Corasaniti, D. Huterer, A. Melchiorri, Phys. Rev. D 75(6), 062001 (2007) 